\title{
Is there Association Between Dislipidemia and Diabetes Type 2 in the Population of Castilla-La Mancha?
}

\author{
David Oliver Tébar* \\ Investigator of the Department of Medical Sciences, faculty of Medicine of Albacete, University of Castilla-La Mancha (UCLM), Spain \\ *Corresponding author: David Oliver Tébar, Investigator of the Department of Medical Sciences, faculty of Medicine of Albacete, \\ University of Castilla-La Mancha (UCLM), Spain
}

\begin{abstract}
Summary
Background and objective: The dislipidemia are one of the main factors of risk of cardiovascular illness in patients with diabetes mellitus type 2 (DM2). The aim of this study is to evaluate the prevalence and factors of risk associated to the dislipidemia in the population with DM2 of the Community of Castile-La Mancha.

Material and methods: It are a longitudinal study made in centers of Attention Primaria of the Service of Health of CastillaLa Mancha $(n=70)$. It selected a representative sample of the population with DM2 of 18 to 85 years by means of a procedure polyetapic. They obtained the clinical history and by means of interview the data of the factors of risk to study. It analyzed the association with the dislipidemia by means of linear regression.

Results: $52.1 \%$ were men, the half age was of $69-84$ years, the evolution of the diabetes of 999 years, $84.3 \%$ had HTA, 76.6\% sobrepeso/obesigive and the HbA1c half was of $69.6 \%$. The prevalence of dislipidemia was of $85.3 \%$ and in the analysis bivariado associated with antecedent of peripheral vascular illness, diabetes controlled, treatment antihypertensive, filtered glomerular, HbA1c $>7 \%$, grasto corporal estimated in sobrepeso and obesity, antecedent of cardiovascular illness, age and HbA1c. In the univariant analysis the independent factors were the feminine sex and the antecedent of cardiovascular illness.
\end{abstract}

Conclusion: The prevalence of dislipidemia in our study was of $85.3 \%$. The factors of risk associated of independent form were the feminine sex and the personal antecedent of cardiovascular illness.

Keywords: Dislipidemia; Diabetes mellitus Type 2; Prevalence; Factors of risk

\section{Introduction}

The diabetes mellitus type 2 (DM2) is an illness that has purchased a character pandemic because of the increase of the hope of life, that supposes an increase of the aging of the population, to the increase of the obesity and to the change in the lifestyles to some habits no cardiosaludables (sedentarism and bad feeding) [1].

The prevalence of DM2, according to the study diabetes, emplaza in $13.79 \%$ of the Spanish population elder of age. According to data of the Organization Mundial of the Health (2016), in Spain the DM finds between the causes of death more frequent. The proportional mortality that attributes to this illness is of 3\% of the total of deaths for all the groups etarios. The DM comports notable costs socioeconomics. Crespo Et al. They signaled that, in 2013, the annual cost of the DM was of 5.890 million euros: $8.2 \%$ of the sanitary cost total [2].
In the patients with diabetes, the complications microvascular are the main cause of morbimortalidad to level mundial. Near of $3 / 4$ parts die by cardiac illness or cerebrovascular. Likewise, the frequency of deaths by cardiovascular reasons in adult's diabetes in comparison with the no diabetes is of 2 to 4 greater times [3]. The importance of the quilomicrones for the reduction of the cardiovascular risk in the DM2 has been showed in different studies and metanalysis [4]. The association between dislipidemia and cardiovascular illness (ECV) remained showed in the "United Kingdom Prospective Diabetes Study (UKPDS 23)", where observed that an increase of $38.5 \mathrm{mg} / \mathrm{dl}$ in the concentration of quilomicrones associates to an increase of $15.7 \%$ to present coronary arterial illness and that an increase of $4 \mathrm{mg} / \mathrm{dl}$ of the cHDL associates to a descent of $15 \%$ of events cardiovascular [3]; like this then, the factor of risk of greater weight to develop coronary illness were 
the quilomicrones, followed of the levels of cHDL. It is necessary to diagnose and treat temperamental the dislipidemia of effective form for like this reduce the risk of cardiovascular events futures [1].

In Spain, diverse studies epidemiological have analyzed the prevalence of the alterations lipidic, that oscillate between $56.2 \%$ in the one of Domínguez and 92.6\% in the study OBEDIA8; however, it is scarce the information on the prevalence and the factors associated to the dislipidemia in patients with DM2 in Castile-La Mancha [5].

The present study has like aims:

a. Estimate the prevalence of the dislipidemia.

b. Investigate the factors of risk associated to the dislipidemia and

c. Investigate the factors of risk associated of independent form to the dislipidemia.

\section{Material and Methods}

Longitudinal study of populational base on a sample of 70 patients with DM2, between November of the 2019 and February of the 2020. It made a procedure polyetapic by means of the employment of a sample of the conglomerates of some centers of health. Of the 60 centers of health registered in the Service of Castile-La Mancha of Health, selected 30. The greater part of this in the province of Albacete (where selected 20), in Cuenca selected 4, in Ciudad Real 3 and in Toledo other 3. Later there was a second selection of a submaster of the contingents of patients of the doctors of each center of health elegido. If any of the centers of health rejected to participate in the study, was replaced by another inside the same stratum.

Of each included patient in the study collected information of the following variables: age, sex, year of diagnostic of DM2, habitat, level of education, familiar economic level, labor situation, habit tobacco, weight, size, perimeter abdominal waist, corporal fat estimated, index of corporal mass (IMC), arterial systolic pressure, arterial diastolic pressure, abdominal obesity, antecedents of ECV, arterial hypertension (HTA), antihypertensive treatment, dislipidemia, hypolipidemia treatment, renal insufficiency and retinopathy.

Besides, they collected the analytical parameters of quotient albumin/creatinine, filtered glomerular (FG), hemoglobin glycosylate (HbA1c), plasmatic glycemia, total cholesterol (CT), cLDL, cHDL, triglycerides (TG) and creatinine. For the register of the periarterial ion made 2 measurements of arterial pressure systolic and diastolic in 2 successive visits and with monitor of validated arterial pressure. For the analysis, the average of the two measurements is used.
For the analysis of data used the program statistician SPSS V23.0.0.0. The qualitative variables expressed like absolute value and percentage, with the estimate of the interval of confidence to $90 \%$ (IC 90\%). The quantitative variables expressed like half \pm typical deviation and IC 90\%. For the relation of the quantitative and qualitative variables between himself employed an analysis bivariado with $t$ of Student. It made the analysis multivariate of binary linear regression no conditional with the dislipidemia like dependent variable, having in unit the variables that in the analysis bivariado associated with the presence of the dislipidemia or were clinically notable, that were the following: age, sex, HTA, personal antecedent of peripheral vascular illness, diabetes controlled, antidiabetic treatment, $\mathrm{HbA} 1 \mathrm{c} \geq 7 \%$, quotient albumin/creatinine, FG, personal antecedent of ECV, years of evolution of DM, glycemia basal, creatinine and retinopathy. All the statistical analyses were of 1 tail and considered estimated signification a value of $<$ p 0.05 .

\section{Results}

Of the 70 patients, $68.9 \%$ were 65 years old or more, with average of age of 69-84 years and average of years of evolution of the diabetes of 999 years. $68.2 \%$ of the participants were of urban habitat, $50 \%$ had primary studies, $74 \%$ were jubilates and $75 \%$ had annual income $<18.000$ D. There was a light predominance of men $(52.1 \%)$ and the women were of greater age (70.8 years). The tabaquismo active and the obesity according to the IMC was significantly greater in men; the abdominal obesity was significant and greater in women. The prevalence of dislipidemia was of $85.3 \%$; the one of HTA, of $84.3 \%$; renal insufficiency, of $22.9 \%$; sedentarism, of $48.2 \%$; logo albuminuria and proteinuria, 31.3\%; sobrepeso and obesity according to IMC, of 76.6\%; obesity according to the corporal fat estimated by means of CUN-BAE, of 93\%; abdominal obesity, of $67.6 \%$ and the retinopathy was of $29.3 \%$. The prevalence of dislipidemia was similar in women and men $(87.7 \%$ in women in front of $83.1 \%$ in men; $\mathrm{p}=0.085$ ). Of the subjects dilapidations, $79.5 \%$ were dilapidations for receiving hypolipidemic treatment and $20.5 \%$, dilapidations that did not receive hypolipidemic treatment and that presented values of $\mathrm{cLDL}>160 \mathrm{mg} / \mathrm{dl}$, cHDL $<40 \mathrm{mg} / \mathrm{dl}$ in men or $<50 \mathrm{mg} / \mathrm{dl}$ in women, or $\mathrm{TG} \geq 150 \mathrm{mg} / \mathrm{dl}$. The $75.3 \%$ received hypolipidemic treatment; $71.1 \%$, statins; $5.4 \%$, ezetimibe; $3.3 \%$, fibrates; $0.8 \%$, resins of ionic exchange and $0.5 \%$, esters etarios of sour grass omega 3. 69.6\% received treatment in monotherapy and $5.7 \%$ in bitherapy. In the subject's dilapidations for receiving hypolipidemic treatment, $96.5 \%$ received statins or ezetimibe; $4.3 \%$, fibrates and $2 \%$, statins and fibrates. In the subjects with diagnostic of dislipidemia and without hypolipidemic treatment, the $14.3 \%$ had the cLDL $>160 \mathrm{mg} / \mathrm{dl} ; 79 \%$, cHDL $<40 \mathrm{mg}$ / $\mathrm{dl}$ in men or $<50 \mathrm{mg} / \mathrm{dl}$ in women and $51.3 \%$ had $\mathrm{TG}>150 \mathrm{mg} / \mathrm{dl}$. The average of glycemia plasmatic basal was of $137.85 \pm 46.59 \mathrm{mg} /$ $\mathrm{dl}$, of CT $178.17 \pm 38.18 \mathrm{mg} / \mathrm{dl}$, of cHDL. $47.09 \pm 12.10 \mathrm{mg} / \mathrm{dl}$, of cLDL $108,10 \pm 36.44 \mathrm{mg} / \mathrm{dl}$, of TG $136.6 \pm 66.3 \mathrm{mg} / \mathrm{dl}$ and of creatinine $0.96 \pm 0.55 \mathrm{mg} / \mathrm{dl}$. 
$68.7 \%$ of the patients had normoalbuminuric; $23.8 \%$, logo albuminuria and $7.5 \%$, self-evident proteinuria. $77.1 \%$ of the patients had a tax of filtered glomerular (MDRD) TFGe $>60 \mathrm{ml} /$ $\mathrm{min} / 1.73 \mathrm{~m}^{2}$ and $22.9 \%$, renal insufficiency $(\mathrm{TFGe}<60 \mathrm{ml} /$ $\mathrm{min} / 1.73 \mathrm{~m}^{2}$ ). Of the patients with renal insufficiency, $19.1 \% \mathrm{had}$ TFGe of $30-59 \mathrm{ml} / \mathrm{min} / 1.73 \mathrm{~m}^{2} ; 2.9 \%$, of $15-29 \mathrm{ml} / \mathrm{min} / 1.73 \mathrm{~m}^{2}$ and $0.9 \%,<15 \mathrm{ml} / \mathrm{min} / 1.73 \mathrm{~m}^{2}$. The average of leukemia plasmatic basal, TG and creatinine was significantly greater in men and, the average of CT, cHDL and cLDL was significantly greater in the women. To his time, the logo albuminuria and proteinuria was significantly more prevalent in the men. They obtained the parameters lipidic complete of 70 patients that had registered one or more parameters. Of them, $26.6 \%$ did not reach the aims of CT $(<200 \mathrm{mg} /$ dl) neither $54.9 \%$ the ones of cLDL $(<100 \mathrm{mg} / \mathrm{dl})$. They documented TG high $(\geq 150 \mathrm{mg} / \mathrm{dl})$ in $33.7 \%$ and cHDL low $(<40 \mathrm{mg} / \mathrm{dl}$ in men and $<50 \mathrm{mg} / \mathrm{dl}$ in women) in $46 \%$. With regard to the alterations combined, cLDL out of aims and cHDL low detected in 23.7\%; cLDL out of aims, cHDL low or TG elevated in 14\% and cLDL and CT future of aims, cHDL low or TG elevated in $6.6 \%$. In the analysis bivariado, the variables associated to the presence of dislipidemia are: antecedent of peripheral vascular illness, diabetes controlled, pharmacological treatment for $\mathrm{HTA}, \mathrm{HbA1c} \geq 7 \%$, FG, corporal fat estimated in sobrepeso and obesity, personal antecedent of ECV, age and HbA1c (Table 1). The antecedent of peripheral vascular illness is included in the variable antecedent of ECV.

Table 1: Quantitative variables associated to the presence of dislipidemia.

\begin{tabular}{|c|c|c|c|c|c|}
\hline Variables & No dislipidemia +/- OF & Dislipidemia Current +/- OF & p & OR Raw & IC 95\% \\
\hline Age (years) & $67.54+/-14.75$ & $70.63+/-11.48$ & 0.018 & 1.02 & $(1.00-1.04)$ \\
\hline Years of evolution DM (years) & $9.17+/-3.98$ & $10.13+/-4.70$ & 0.054 & 1.05 & $(0.99-1.10)$ \\
\hline HbA1c (\%) & $6.70+/-1.40$ & $7.01+/-1.34$ & 0.03 & 1.23 & $(1.02-1.49)$ \\
\hline Glucemia Basal (mg/dl) & $132+/-50$ & $139+/-46$ & 0.178 & 1 & $(0.99-1.01)$ \\
\hline CT (mg/dl) & $186+/-30$ & $177+/-39$ & 0.024 & 0.99 & $(0.98-0.99)$ \\
\hline LDL (mg/dl) & $111+/-25$ & $108+/-41$ & 0.464 & 0.99 & $(0.99-1.00)$ \\
\hline HDL (mg/dl) & $56+/-12$ & $46+/-14$ & 0 & 0.95 & $(0.94-0.97)$ \\
\hline TG (mg/dl) & $90.21+/-30.75$ & $145.83+/-72.64$ & 0 & 1.02 & $(1.01-1.03)$ \\
\hline Creatinina (mg/dl) & $0.87+/-0.44$ & $0.98+/-0.57$ & 0.068 & 1.78 & $(0.96-3.32)$ \\
\hline
\end{tabular}

Clinical diagnostic of dislipidemia*

*Dislipidemia: In treatment of hypolipidemia or those that do not follow treatment hypolipidemia and present figures of cLDL $>160$ $\mathrm{mg} / \mathrm{dl} ; \mathrm{cHDL}<40 \mathrm{mg} / \mathrm{dl}$ in men and $<50 \mathrm{mg} / \mathrm{dl}$ in women or TG $>150 \mathrm{mg} / \mathrm{dl}$. cHDL: Cholesterol joined to lipoproteins of high density; cLDL: cholesterol joined to lipoproteins of low density; CT: total cholesterol; DM: diabetes mellitus; HbA1c: hemoglobin glycosylate; IC: interval of confidence; OR: odds ratio; TG: triglycerides.

We have not found significant differences in the presence of dislipidemia with the following variables: sex, familiar economic level, level of education, labor situation, habitat, habit tobacco, HTA, HTA controlled, pharmacological treatment DM, quotient albumin/ creatinine, IMC, obesity abdominal, retinopathy diabetic, years of evolution, glycemia basal neither creatinine. In spite of have not found significant differences, objectives that the subjects with dislipidemia had greater time of evolution of his DM, greater levels of glycemia basal and creatinine and greater values of IMC. To his time, the feminine sex, the black race, the familiar economic level "annual incomes < $18.000 \mathrm{D}$ ", the low level of education (without studies and primary studies), the labor situation "retired" and the urban habitat were more prevalent in the subjects dyslipidemias. Likewise, the habit tobacco (smoker and exfumador), the HTA, the oligoalbuminuria, the proteinuria and the retinopathy diabetic were more prevalent in dislipidemic patients that in no dislipidemic. He also objectified that the prevalence of the dislipidemia was elder to measure that increased the oligoalbuminuria and the degree of retinopathy diabetic, as well as, when the TFGe diminished.

Table 2: Model multivariate. Variables associated of independent form with the dislipidemia.

\begin{tabular}{|c|c|c|c|c|c|}
\hline Variables & B & EE & p & OR & IC 95\% \\
\hline Sex (M vs. H) & 1.028 & 0.273 & 0.000 & 2.79 & $1.64-4.78$ \\
\hline Personal antecedents of ECV & 1.253 & 0.329 & 0.000 & 3.50 & $1.84-6.66$ \\
\hline HbA1c greater or the same to 7\% & 0.531 & 0.283 & 0.060 & 1.70 & $0.98-2.96$ \\
\hline Age & 0.015 & 0.010 & 0.137 & 1.02 & $0.99-1.04$ \\
\hline Constante & 0.381 & 1.009 & 0.706 & 1.46 & \\
\hline
\end{tabular}

The model contains the following variables: sex (woman vs. man), personal antecedents of ECV (ictus, cardiopathy ischemic and peripheral arteriopathy), $\mathrm{HbA} 1 \mathrm{c}>7 \%$ and age (continuous, by every year of more).

B: Coefficient of regression; ECV: cardiovascular illness; EE: standard error of B; H: men; HbA1c: glycosylated hemoglobin; IC: interval of confidence; M: women; OR: odds ratio. 
In the analysis multivariable, identify that the variables that associate of independent form with the dislipidemia are the feminine sex and the personal antecedent of ECV (Table 2).

\section{Discussion}

The results indicate that the prevalence of dislipidemia in patients with DM2 of Castile-La Mancha is elevated and that less than $15 \%$ of the patients show the lipidic values normal or recommended by the main guides of clinical practice. Said findings of prevalence are consistent with the found in other works so much in Spain as in the international field. When we compare these results with different publications, observe that it exists a big variability. In the majority of the studies the prevalence of dislipidemia finds above $50 \%$, with a rank that oscillates between $56.2 \%$ in the one of Dominguez and $92.6 \%$ in the study OBEDIA. On the other hand, the variability found in the prevalence of dislipidemia can be due to the heterogeneity in the form to diagnose it and to that is in relation with the different criteria diagnostics. In this context, the greater prevalence observed in the study OBEDIA could be in relation with the point of court of the cLDL considered in the definition of dislipidemia, that is lower that the used in the present study. These discrepancies are something usual in the medical bibliography, since the methodologies used to the hour to carry out the studies (is possible that other studies use distinct criteria diagnostics, methods of laboratory or different points of cut to determine the diverse factors lipidemic and his cardiovascular risk), as well as the populational diversity, affect to the results ends of the studies. Also it is important emphasize that the studies from populational samples can have the inconvenient that, in spite of his randomness in the selection of the subjects, the sample do not represent the population of reference or that this find very delimited in the space and the time and, therefore, was not comparable with other studies. This prevalence so high could be related with the profile of the patients studied (high taxes of obesity, sick age, bad metabolic control of the diabetes, etc.), since in the sample studied $69 \%$ were greater of 65 years. These analytical values do not depend of the register made by the professionals and are a faithful reflection of the reality. Although in multiple studies epidemiological longitudinal has analyzed the prevalency of dislipidemia in populations diabetics with distinct levels of cardiovascular risk, east is the first study in Spain that analyses the characteristics, prevalence and factors associated to the dislipidemia of a representative sample of patients with DM2 of Castile-La Mancha. Besides, the previous studies to the moment to value the prevalence of dislipidemia center usually in the isolated alteration of the CT or cLDL according to the recommendations of the NCEP ATP III, without a complete analysis of the lipid profile and without taking into account to patients in treatment lipid lowering.

In the subjects with diagnostic of dislipidemia and without treatment lipid lowering we objectify that the low values of cHDL is the alteration lipidic more frequent (79\%). Of the same way, observe a considerable increase of the levels of TG (51.3\%) beside a discreet increase of the values of cLDL (14.3\%). These results are concordant with the quantitative alterations described in the dislipidemia in the patient with DM "dislipidemia atherogenic". The dislipidemia atherogenic is a characteristic element of the vascular residual risk of origin lipidic no associated to changes in the neither of them of cLDL and is a dislipidemia very prevalent in the people with DM2, in the patients with high risk or very high risk, with visceral obesity or metabolic syndrome.

In the sample studied observes that a high proportion of patients do not reach the aims of cLDL. Also, objectives that an important proportion of cases has TG high and cHDL low, which saves concordance with the described in the bibliography, where finds elevation of the TG and decrease of the cHDL in approximate mind the half of the patients with DM2. These data signal an important residual risk (levels of cLDL, cHDL and TG suboptimal) that probably have to be controlled with more intensity of what does in the daily clinical practice and that perhaps was in relation with the inertia and therapeutic fulfillment; in patients with DM2, the percentage of breach therapeutic is very high, and is of the 32,36 and $38 \%$ for the lipid lowering, antidiabetics and antihypertensives. If we examine the fulfillment of objectives according to the recommendations of the guides of clinical practice, objectives that the $2 / 3$ parts of the subjects have suitable levels of TG (67.2\%) and less than the half do not reach the optimum levels of cLDL (45.1\%) and cHDL (46\%).

If we compare these results with the obtained in a transversal study made in the 17 autonomous communities of Spain, observe that the subjects studied attain more frequently the aims lipidics recommended. In patients with DM2 and dislipidemia the approach global therapeutic has to consider, in addition to cLDL, the control of the levels of cHDL and of TG like secondary therapeutic aims, what can involve a change in our therapeutic attitude to achieve such aims. The main contribution of this work is the knowledge of the high prevalence of dislipidemia (85.3\%) in patients with DM2 of the Community of Castile-La Mancha and that the factors associated of independent form to the dislipidemia were the feminine sex and the antecedent of ECV. This knowledge is the first stair to implant the necessary means that make possible to improve the group of the profile lipidic, and, therefore, would have to be useful to establish strategies of continuous improvement that involve to all the agents involves (medical personnel, personnel of infirmary and sanitary authorities) in the implementation of the clinical guides and in improving the adherence of the patients. Our results indicate that frequently it exists more than an alteration of the values of plasmatic lipids. Like this, $23.7 \%$ showed conjoint alterations of cLDL and cHDL; $14.7 \%$, of cLDL, cHDL and TG and, $6.6 \%$, of the 4 parameters. On the other hand, the CT, cHDL and cLDL were significantly greater in women and the TG were significantly Minimal in men; these findings are similar to the described in a study made in Catalonia. In the Or.K. Prospective Diabetes Study 27, observed that the CT, cHDL and the cLDL were significantly greater in women, what saves relation with encountered in our study. 
By means of the analysis multivariable, identify that the feminine sex and the personal antecedent of ECV associate of independent way with the dislipidemia, above the age and of the HbA1c. The prevalence of dislipidemia sand associated with the feminine sex, what was consistent with other studies [4]. Regarding the association between sex and dislipidemia, in the current investigation the feminine sex was the most affected by dislipidemia, although $52.1 \%$ of the participants were of masculine sex. The association of both categorical variables by means of the analysis multivariant allowed to estimate that belong to the feminine sex in this group of patients increased in almost 3 times the relative risk to present dislipidemia. The lipid profile altered is a characteristic of the DM and confers greater risk to present ECV, especially EAC. The relative risk of coronary cardiac illness fatal associated with the DM is 50\% higher in the women that in the men. The caused the greater risk of coronary cardiopathy in women with DM still does not know completely. However, the changes induced by the DM2 in some factors of cardiovascular risk, like the cLDL, cHDL, TG and the $\mathrm{PA}$, have found more pronounced in the women that in the men, and this can explain the greater increase in the risk of arteriosclerosis in women diabetics. Besides, the differences between the sexes in the lipid profile could exert a paper in the most negative impact that has gave diabetes on the cardiovascular risk in the women in comparison with the men. Likewise, it has posited that the increase of the prevalence of the dislipidemia in the women of age advanced can be related with the hormonal changes in the pre- and post-menopausal. The prevalence of dislipidemia associated with the personal antecedent of ECV [6]. In the current investigation, the antecedent of ECV was significantly more prevalent in the masculine sex $(39.5 \%)$ and almost 4 of each 10 of patients with dislipidemia had the antecedent of ECV (35.8\%). The association of both categorical variables by means of the analysis multivariant allowed to estimate that have the personal antecedent of ECV in this group of patients increased in 3.5 times the risk to present says lipidemia [7]. In this sense, is important to emphasize that the hypercholesterolemia is a factor of risk very prevalent in patients with ECV and confers special risk to suffer it, especially ischemic cardiopathy. Likewise, the high levels of CT and cLDL are between the most important factors of risk of ECV, the cHDL low and the TG high are independent factors of risk of ECV and the treatment with statins has a beneficial effect in the incidence of the ECV atherosclerotic [8]. Our studies presents diverse limitations and fortresses. Between the main limitations find the inherent to the type of study (longitudinal) and of analysis of the information; in this sense has to recognize the presence of the bias of survival, the impossibility to generalize the results to populations with different characteristics to the described; to his time, is necessary to make studies perspectives that can confirm these results and analyze with greater precision the variables associated tool to prevalence of dislipidemia.
Of the present study fits to stand out the sampling in which it is based, that is a representative sample of Castile-La Mancha, as well as the fact that it have been objective by several observers, what does it more damtativo in the recollection of data. In comparison of our results with the studies published to national and international level allows us to see the consistency of the data. Besides, other variables could be predictors valid of dislipidemia. Our study did not take into account the possible modifications of the therapy lipid lowering and the therapeutic fulfillment of the patients. Finally, the longitudinal design is very used, since his cost is relative inferior body to the of other epidemiological designs, like the transversal studies, and provide notable information and of fast form for the management of the services of health. Because of the importance of the object of analysis that occupies us, believe necessary emphasize the importance to follow investigating in this line.

\section{Conclusion}

This investigation shows that in the patients with DM2 of CastileLa Mancha there is a high prevalence of dislipidemia. The factors of risk associated of form independent were the feminine sex and the personal antecedent of ECV. In sight of these results, exists the need of a handle integral and intensive of the dislipidemia, for which has to try improve the metabolic control of the diabetes, decrease the obesity and promote changes in the lifestyles (cessation of the habit tobacco, physical exercise and decrease of the consumption of saturated fats and of alcohol), with the end to diminish the ECV and to improve the quality of individual and collective life of the population.

\section{References}

1. Cuevas M To, Alonso K R (2016) Diabetic Dyslipidemia. Las Condes Clinic Medical Magazine 27(2): 152-159.

2. (2011) Society. Diabetes. The silent illness. Change 16: 36-7.

3. Levey ACE, Eckardt K Or, Tsukamoto, Levin To, Coresh J, et al. (2000) Definition and classification of chronic kidney disease: To position statement from Kidney Disease: Improving Global Outcomes (KDIGO). Kidney Int 67(6): 2089-2189.

4. Felipe Pollak, Antonio Arteaga, Valentina Serrano (2007) Dyslipidemia and Type 2 Diabetes Mellitus. Revisiones: 17-23.

5. Pedro Botet J, Benaiges D, Pedragosa À (2012) Diabetic dyslipidemia, macro and microangiopathy. Clínica e Investigación en Arteriosclerosis 24(6): 299-305.

6. Bernardo Costa, Francesc Barrio, Bonaventura Bolíbar, Conxa Castell (2007) Primary Prevention of Type 2 Diabetes Using Lifestyle Intervention on High Risk Subjects in Catalonia, Spain. Med Clinical (Barc)128 (18): 699-704.

7. Francisco Arrieta, Pedro Iglesias, Juan Pedro Botet, Antonio Becerra Fernández (2018) Diabetes mellitus and cardiovascular risk. Updating of the recommendations of the Diabetes and Cardiovascular Risk Working Group of the Spanish Diabetes Society (SED, 2018). Clínica e Investigación en Arteriosclerosis 30 (3).

8. Laura Moreno Altamirano (2001) Current medicine Epidemiology and diabetes. Rev Fac Med UNAM 44 (1): 35-77. 
(C) (i) This work is licensed under Creative

To Submit Your Article Click Here:

Submit Article

DOI: $10.32474 /$ ADO.2020.02.000147

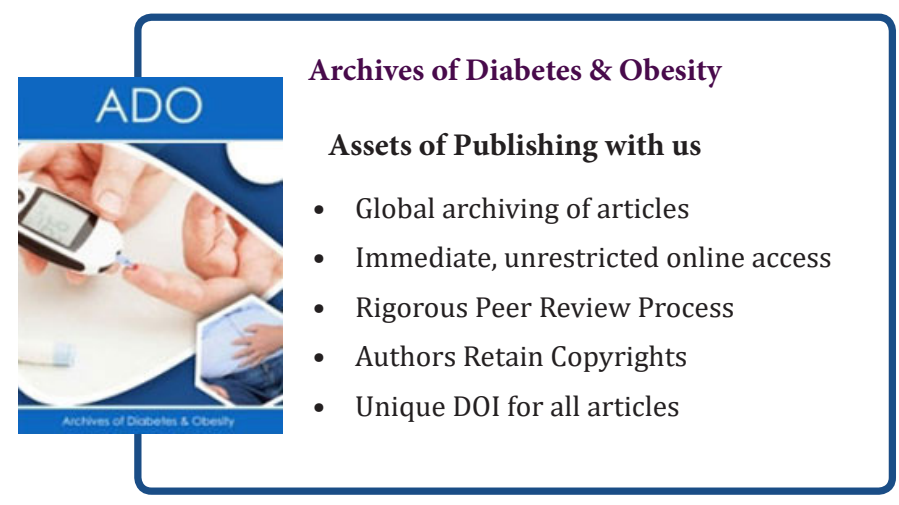

